\title{
Algorithm of wireless sensor network node based on the vehicle
}

\section{localization}

\section{FAN HENG}

\section{JILIN ENGINEERING NORMAL UNIVERSITY}

\begin{abstract}
Keywords: Wireless, Node. algorithm
\end{abstract}
Abstract: This article is presented based on the wireless sensor node design and algorithm of vehicle tracking localization of research, the use of wireless sensor network with low cost, low power consumption, multi-function, the characteristics of the sensor nodes deployed in monitoring area, through the vehicle's sound, vibration, infrared, magnetic field, such as signal analysis, to identify location and tracking, and in appropriate time to continue to track vehicles.

\section{Introduction}

Wireless sensor network (WSN) is mainly used to monitor various environmental features in network deployment area, such as temperature, humidity and luminosity, sound intensity and magnetic field intensity, the pressure, the acceleration of a moving object/speed/pressure, concentration of chemicals and so on.But for the sensor data in the case of don't know the corresponding location information, often does not make sense. That is to say, the location of sensor nodes in wireless sensor network (WSN) plays an important role in many application fields of.In vehicle tracking applications, the target node to perceive movement of speed and position of the node, can monitor the target movement route and predict the movement direction of the target.Node location information can also help for the design of the other protocol layer, the application layer, the node location information to choose a location-based information service application is also indispensable.Through gathering more data for energy conservation of sensor nodes, the location information is also very important.In network layer, the combination of location information and transmission distance, make it possible to routing algorithm based on geographic location routing strategy based on node location information can more effectively through multiple hops to spread information in the wireless sensor network (WSN), these typical protocols including DV - Hop algorithm, TBF routing algorithm, SPEED real-time communication protocol, LAP scalable routing protocol and energy efficient routing method.This paper research on the positioning system of vehicle tracking using DV - Hop algorithm for the realization of the non-contact ranging localization algorithm.

\section{The characteristics of wireless sensor network localization algorithm}

How do you determine the node localization in wireless sensor networks become key problems that must solve.Node localization namely so-called by a certain technology, method and means for wireless sensor network node in the process of absolute or relative position information.Due to the node hardware configuration is low, the energy, computing, storage and communication ability is limited, so the node location puts forward the challenge, the characteristics of wireless sensor network localization algorithm generally meet the following properties 


\section{System architecture}

Vehicle tracking system based on wireless sensor network is composed of four layers structure: wireless sensor network (WSN), base station, the server and client.By an on-board wireless sensor nodes and testing nodes Vehicular node is installed on the vehicle active communication device, periodically sends its own information, for detecting node detection Detection node arrangement of distributed on both sides of highway network, detection of vehicle information, after dealing with the data through multiple hops forward testing information will be sent to the base station, a base station receive vehicle information from the sensor network, data fusion, send the result to the Internet The server from the Internet to get the data, the database record and query and provide services The client via cable or wireless network server, query the vehicle information, vehicle trajectory Overall system structure as shown

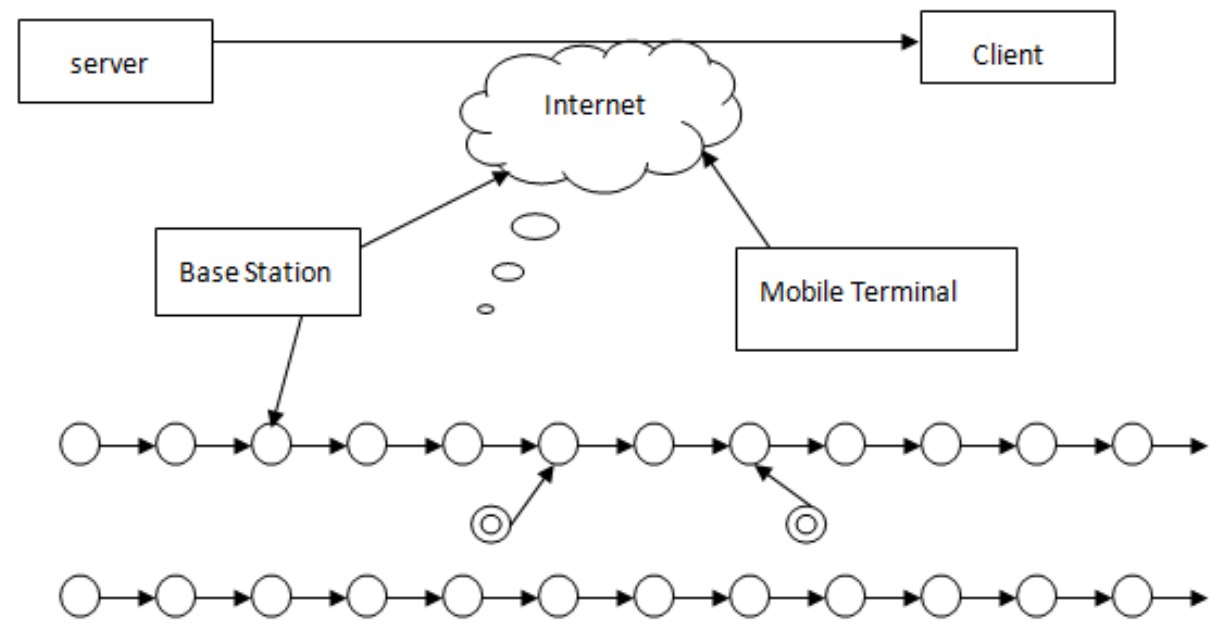

Figure 1 The structure of vehicle tracking system

\section{DV - Hop algorithm and related algorithms}

The localization algorithm of this system USES the DV - Hop algorithm, this algorithm according to the principle of distance vector routing is a series of distributed localization algorithm is put forward DV - Hop algorithm is the core idea of the unknown node through the routing method "measure" and the leading mark minimum Hop count between nodes, and calculate the average every jump distance, average with the least Hop and jump from the product of the estimate as the distance between the unknown node and beacon node

DV - Hop algorithm of localization process can be divided into three stages

(1) measurement and leading marks the minimum hop count between nodes

Using typical exchange of distance vector routing protocol, leading mark radio beacon node to the neighbor node information, including location information and hop Location information of nodes record received this information, and the hop and forwarding its neighbor nodes, so that all the nodes in the network to be able to record each beacon node location and the corresponding minimum hop count In order to reduce network traffic, nodes can be discarded "invalid" beacon node information. If a beacon node information has been received and the corresponding number of not less than the minimum hop of records, the information is considered to be invalid.

(2) estimated distance calculation and beacon nodes

Beacon nodes according to the records in the first phase of the position information of other beacon nodes and the corresponding minimum hop count, according to the type (1) estimate the average distance of each jump 


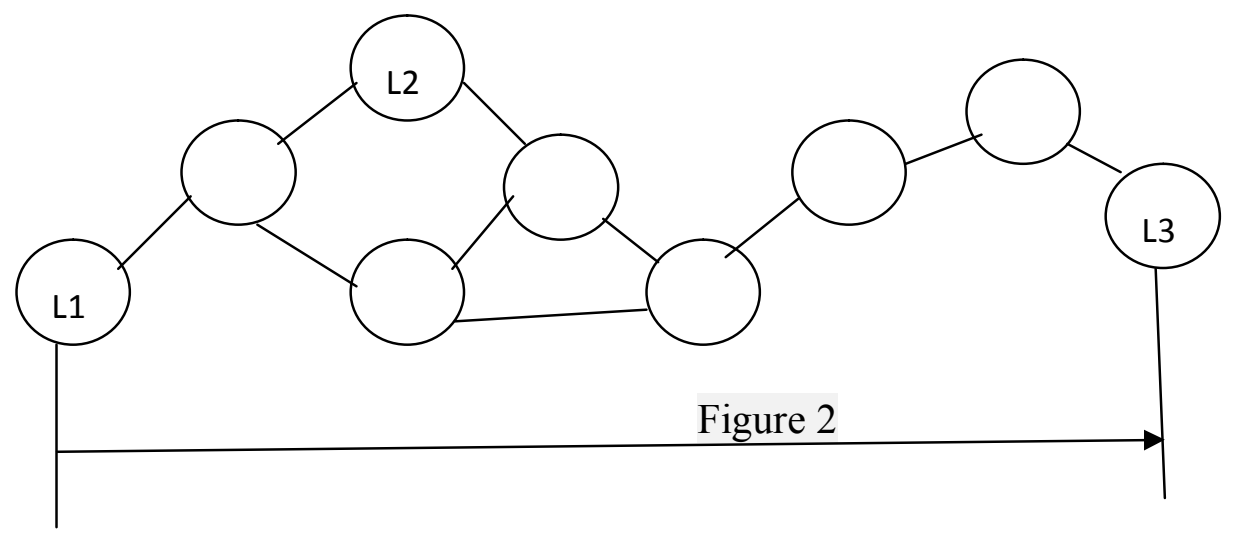

As shown in figure 2, L1, L2 and L3 are beacon nodes, L1 can compute the distance of L2 and L3, and the corresponding minimum hop dance by 2 and 6, respectively and L1 calculated an average jump distance of $17.5 \mathrm{~m}$.L2 and L3 also calculate the average jump distance of $16.42 \mathrm{~m}$ and 15.90 $m$ respectively Then $\mathrm{L} 1$ broadcast the average hop distance value, the unknown node after receiving forwarded to its neighbor nodes, and according to the records of the minimum hop estimation and the distance between the beacon nodes In order to avoid duplication of receiving the same beacon node average jump distance information, can be used in a controllable flood routing method When the network scale, according to the above methods will receive from leading mark too much unknown node average jump from information, and time delay is big, DV - Hop algorithm into an average jump distance information lifecycle TTL control to solve the problem.

(3) Estimate the unknown node location

By the second phase of the unknown node estimated distance of each beacon nodes according to the measurement or maximum likelihood estimation method to calculate the coordinates. Simulation experiments show that the DV - Hop algorithm in network connection degree an average of 9, beacon node ratio of $10 \%$ under isotropic network configuration, positioning accuracy is approximately $33 \%$, the shortage of the DV - Hop algorithm is only performed well in isotropic network performance, because only in the isotropic network average jump distance to reasonably estimate the actual distance between the nodes.

\section{The conclusion}

Vehicle tracking system of wireless sensor network (WSN) this paper puts forward the design scheme of target detection, network communication, based on the system architecture to determine the sensor node design, hardware design, the design of communication protocol, for the establishment of the vehicle tracking system to build solid theoretical basis Adopting modular design, the use of high performance and high scalability JN5121 chip, the scheme has good versatility and high reliability In the vehicle positioning monitoring system, has the very strong practicability and convenience, and has certain promotion value.

\section{References}

[1] Wang Shu Yan Yujie, the theory and application of wireless sensor network (WSN), 402-403 (2007).Reference to a book:

[2] Ren Shansi,Li Qun,Wang Haining. Design and Analysis of Sensor Seheduhng Algorithms Under Partial Coverage for Object Detection in Sensor Works[J].IEEE Transactions on Parallel and Distributed Systems,2007,(03):334-350. 
[3] kuangxinghong,Location tracking technology in wireless sensor network (WSN) research. Shanghai jiaotong university in 2008 to join collection Get the latest

[4] J.Rayson.Aggregate Towers:Scale Sensitive Visualization and Declutteriong of Geospacial Data .IEEE, 1999.

[5] N.Ramanathan,E.Koher,D.Estrin.Towards a debugging system for sensor network.International Journal for Network Managenment, Jul2005, Volume 15,Issue 4,223-224 